\title{
Macrocephaly-developmental delay syndrome
}

INSERM

\section{Source}

INSERM. (1999). Orphanet: an online rare disease and orphan drug data base.

Macrocephaly-developmental delay syndrome. ORPHA:397612

Macrocephaly-developmental delay syndrome is a rare, intellectual disability syndrome characterized by macrocephaly, mild dysmorphic features (frontal bossing, long face, hooded eye lids with small, downslanting palpebral fissures, broad nasal bridge, and prominent chin), global neurodevelopmental delay, behavioral abnormalities (e.g. anxiety, stereotyped movements) and absence or generalized tonic-clonic seizures. Additional features reported in some patients include craniosynostosis, fifth finger clinodactyly, recurrent pneumonia, and hepatosplenomegaly. 